\title{
Potencial en el uso de las propiedades químicas como indicadores de calidad de suelo. Una revisión
}

\section{Potential for the use of chemical properties as indicators of soil quality. A review}

JOAN SEBASTIÁN GUTIÉRREZ D.1, 4

WILLIAM ANDRÉS CARDONA²

OSCAR IVÁN MONSALVE C. ${ }^{3}$

Determinación de la calidad del suelo mediante indicadores químicos en naranja Valencia.

Foto: H. Velásquez

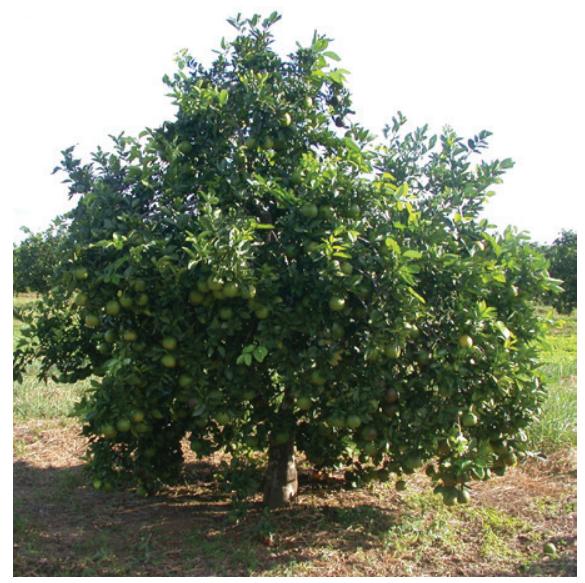

\section{RESUMEN}

La necesidad de entender y evaluar la calidad de los suelos en sistemas de productivos agrícolas es un tema de creciente importancia en la actualidad, debido al interés sobre la determinación de los impactos de las distintas prácticas de manejo en la sostenibilidad del recurso suelo. El objetivo de esta revisión es brindar un panorama general sobre el concepto de calidad de suelo, indicadores para su evaluación y exponer algunas investigaciones realizadas con respecto al potencial de las propiedades químicas para ser usadas como indicadores de calidad de suelos. Entre las propiedades químicas que pueden considerarse como indicadores se encuentran la disponibilidad de nutrimentos, el contenido de carbono orgánico total y carbono orgánico lábil, el pH, la capacidad de fijación de fosfatos, la conductividad eléctrica, la capacidad de intercambio catiónico, contenido de nitrógeno total y mineralizable, y de materia orgánica. Se resalta la importancia de las técnicas multivariantes para definir aquellas propiedades químicas con potencial para ser indicadores de calidad de suelo. La creciente preocupación sobre el uso sostenible del recurso suelo, genera la necesidad de desarrollar enfoques y herramientas que permitan generar diagnósticos acerca de los efectos que tienen las prácticas de manejo implementadas en los sistemas de producción agrícola, es decir, que brinden información acerca de la calidad del suelo en un determinado contexto.

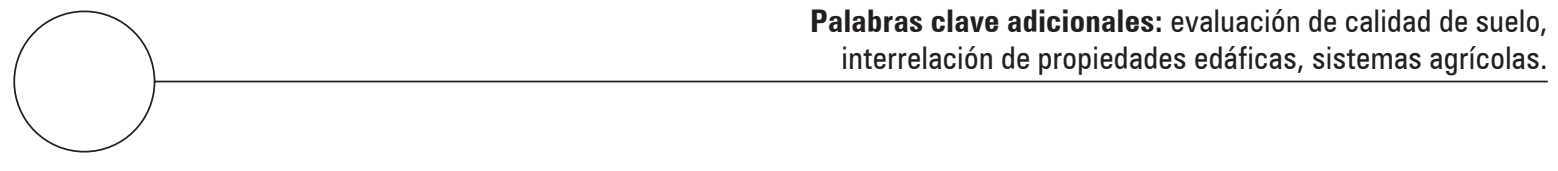

Programa de Maestría en Ciencias Agrarias, Línea Suelos y Aguas, Universidad Nacional de Colombia, Bogotá (Colombia). ORCID Gutiérrez D., J.S.: 0000-0001-6052-9588

2 Corporación Colombiana de Investigación Agropecuaria (Corpoica), Mosquera (Colombia). ORCID Cardona, W.A.: 0000-0001-9610-4135

3 Programa de Doctorado en Ciencias Agrarias, Línea Suelos y Aguas, Facultad de Ciencias Agrarias, Universidad Nacional de Colombia, Bogotá (Colombia). ORCID Monsalve C., O.I.: 0000-0003-2302-805X

4 Autor de correspondencia. jsgutierrezd@unal.edu.co 


\section{ABSTRACT}

The need to understand and evaluate the quality of soils in agricultural production systems is an issue of increasing importance today because of the determination of impacts from management practices on the sustainability of this resource. The objective of this review is to provide an overview of the concept of soil quality and indicators for its evaluation and to present some research on the potential of chemical properties for use as indicators of soil quality. Among the chemical properties that can be considered as indicators are nutrient availability, organic carbon content and labile organic carbon, $\mathrm{pH}$, phosphate binding capacity, electrical conductivity, cation exchange capacity, total nitrogen and mineralizable and organic matter. The importance of multivariate techniques to define chemical properties with a potential as soil quality indicators is highlighted. The greatest concern for the sustainable use of soil resources includes the need to develop approaches and tools that generate diagnoses for the effects of management practices implemented in the systems of agricultural production, that is, to provide information about soil quality in a given context.

Additional key words: soil quality assessment, interrelation of soil properties, agricultural systems.

Fecha de recepción: 02-01-2017 Aprobado para publicación: 30-09-2017

\section{INTRODUCCIÓN}

Como se sugirió a principios de los años noventa, la calidad de suelo es "la capacidad de un suelo para funcionar dentro de un contexto determinado" (De la Rosa y Sobral, 2008). Larson y Pierce (1994) definieron la calidad de suelo como la capacidad del mismo para funcionar dentro de los límites de un ecosistema y para interaccionar positivamente con ecosistemas circundantes. Karlen et al. (1997) definieron este concepto como la capacidad de un tipo específico de suelo para funcionar dentro de los límites de un ecosistema natural o intervenido, con el fin de sostener la productividad animal y vegetal, mantener y aumentar la calidad del agua y aire, y soportar las actividades antropogénicas.

El concepto de calidad de suelo está asociado a dos factores fundamentales: las propiedades inherentes y dinámicas del suelo (Karlen et al., 2003), y la evaluación de la calidad misma que se da en función del uso y vocación del suelo (Khormali y Shamsi, 2009). De ahí que, para que este concepto sea utilizable es necesario contar con variables que puedan ser usadas para evaluar la condición del suelo, surgiendo así los indicadores, como parámetros que representan una condición actual o brindan información acerca de cambios o tendencias en la calidad del suelo (Cruz et al., 2004). Por lo anterior, un indicador es un atributo simple de suelo que puede ser medido para evaluar la calidad, con respecto a una función determinada. Por tanto, es importante seleccionar los atributos apropiados, dada la naturaleza compleja del suelo y el gran número de parámetros que pueden ser determinados en este (De la Rosa y Sobral, 2008).

Según Cantú et al. (2007) un indicador es una variable que resume o simplifica información relevante, y que cuantifica, mide y comunica, en forma comprensible dicha información, de tal forma, que haga perceptible un fenómeno o condición de interés. Los indicadores deben ser preferiblemente variables cuantitativas, aunque también pueden ser cualitativas o nominales u ordinales, especialmente cuando no se dispone de información cuantitativa, o el atributo no es susceptible a ser cuantificado.

Particularmente, los indicadores de calidad de suelo son considerados como herramientas de medición que deben ofrecer información sobre sus propiedades del suelo, procesos que allí ocurren y características generales del mismo. Estos son medidos para dar seguimiento a los efectos del manejo sobre el funcionamiento del suelo en un periodo dado (Astier et al., 2002; García et al., 2012).

De acuerdo con Hünnemeyer et al. (1997), mediante el uso de indicadores de calidad, debe ser posible: (I) Analizar la situación actual del suelo e identificar los puntos críticos con respecto a su uso sostenible; (II) 
Identificar posibles impactos antes de una intervención en un contexto determinado; (III) Monitorear el efecto de las intervenciones antrópicas sobre el recurso suelo y (IV) Ayudar a determinar si el uso del recurso suelo es sostenible. En vista de la importancia de los indicadores como herramientas funcionales para determinar la calidad del suelo en condiciones naturales o intervenidas, es necesario precisar el uso de propiedades que permitan estimar la condición del suelo en condiciones específicas. Dentro de este contexto, esta revisión tiene como propósito brindar un panorama general sobre indicadores de calidad de suelo y exponer algunas investigaciones realizadas con respecto al potencial de las propiedades químicas para ser usadas como tal. Adicionalmente, se busca presentar algunas interrelaciones entre propiedades químicas y biológicas que en conjunto pueden ser usadas para determinar la calidad de los suelos.

\section{PROPIEDADES QUÍMICAS COMO INDICADORES DE CALIDAD DE SUELOS}

Los indicadores químicos de calidad de suelo se refieren a las condiciones de este tipo, que afectan las relaciones suelo-planta, la reserva y calidad del agua, la capacidad amortiguadora del suelo, y la disponibilidad de agua y nutrimentos para las plantas y los microorganismos (SOI, 1996). Entre los indicadores químicos de calidad de suelo se encuentran comúnmente la disponibilidad de nutrimentos, el contenido de carbono orgánico total y carbono orgánico lábil, el $\mathrm{pH}$, la capacidad de fijación de fosfatos, la conductividad eléctrica, la capacidad de intercambio catiónico, contenido de nitrógeno total y mineralizable y de materia orgánica (Cruz et al., 2004).

Tanto las propiedades físicas como químicas, han sido usadas ampliamente para evaluar la calidad de los suelos (Parry Papendick, 1997). No obstante, estas propiedades cambian usualmente dentro de escalas de tiempo (décadas) que son demasiado extensas para propósitos de analizar calidad de suelos dentro del contexto de las prácticas de manejo de este recurso (García-Ruiz et al., 2009).

Las propiedades biológicas son más dinámicas y, por tanto, tienen la ventaja de servir como señales tempranas de degradación o agradación de los suelos. No obstante, hay indicadores que requieren de más de 10 años para mostrar cambios como respuesta a un manejo determinado (Tab. 1).

\section{Interdependencia de indicadores de calidad de suelo}

Además de la escala de tiempo en la que se modifican las propiedades de los suelos, es importante considerar la interconexión entre los parámetros químicos y biológicos en los suelos. Según Schoenholtz et al. (2000) resulta complejo separar las funciones del suelo en procesos químicos, físicos y biológicos, dada su naturaleza dinámica e interactiva, como consecuencia se tiene, por ejemplo, que el contenido de nitrógeno mineralizable pueda ser contemplado como un indicador químico y microbiológico. En suma, algunas propiedades químicas del suelo influyen directamente sobre procesos microbiológicos, y estos procesos en conjunto con aspectos físicos, determinan la capacidad del suelo para mantener, suministrar y ciclar nutrientes y para el movimiento y disponibilidad de agua. Chappel et al. (1999) estudiaron variaciones en la estabilidad de agregados en Ultisoles de Indonesia, disturbados por varios procesos erosivos. Las diferencias en la estabilidad de agregados se correlacionaron con contenido de carbono orgánico, contenido de arcilla y el porcentaje de sodio intercambiable. El carbono orgánico fue el factor más importante, explicando $56 \%$ de la variación en la estabilidad de agregados, lo cual ha sido demostrado por Franzlubbers y Arshad (1996) y Arshad y Martin (2002).

\section{Evaluación de calidad de suelo}

Básicamente se reportan dos tipos de enfoques empleados para la evaluación de la calidad de los suelos: el primero es la evaluación comparativa y el segundo la evaluación dinámica (Larson y Pierce, 1994). En las evaluaciones comparativas, se han utilizado análisis estadísticos multivariantes para identificar indicadores e interpretar correlaciones entre los mismos. Estos estudios en su mayoría, se basan en el análisis de diferentes usos de suelo dentro de un grupo dominante de tipo de suelo (Brejda et al., 2000) o en suelos con el mismo uso, pero bajo diferentes esquemas de manejo Govaerts et al. (2006). Con relación al enfoque dinámico, su fundamento es monitorear los cambios en indicadores claves bajo un esquema de manejo determinado, definiendo de esta manera si la calidad de suelo aumenta, se mantiene o disminuye (Lal, 1998; Karlen et al., 2008).

Distintas investigaciones sobre evaluación de calidad de suelo se han realizado bajo diferentes condiciones 


\begin{tabular}{|c|c|c|c|c|c|}
\hline Indicador & $\begin{array}{l}\text { Profundidad } \\
\text { (cm) }\end{array}$ & $\begin{array}{l}\text { Tipo de } \\
\text { tendencia }\end{array}$ & $\begin{array}{c}\text { Tiempo requerido para } \\
\text { percibir cambios }\end{array}$ & $\begin{array}{l}\text { Frecuencia de } \\
\text { medición }\end{array}$ & Referencia \\
\hline \multicolumn{6}{|l|}{ Indicadores físicos } \\
\hline Textura (\% de arena, limo y arcilla) & $0-30$ & Equilibrio & $>10^{3}$ años & Cada 2-3 años & Arnold et al., 1990 \\
\hline Densidad aparente $\left(\mathrm{g} \mathrm{cm}^{-3}\right)$ & $0-7,5$ & Reducción & $<10$ años & Anual & Arshad y Coen, 1992 \\
\hline Tasa de infiltración (cm min³) & $0-15$ & Incremento & $<1$ año & Periódicamente & $\begin{array}{l}\text { Arnold et al., } 1990 \\
\text { Doran et al., } 1994\end{array}$ \\
\hline Retención de humedad (\%) & $0-15$ & Incremento & $<1$ año & Estacional & Arnold et al., 1990 \\
\hline Resistencia a la penetración (MPa) & $0-50$ & Disminución & $<1$ año & Estacional & Arnold et al., 1990 \\
\hline Profundidad de capa arable (cm) & $0-100$ & Incremento & $>10$ años & Anual & $\begin{array}{l}\text { Arshad y Coen, } 1992 \\
\text { Doran et al., } 1994\end{array}$ \\
\hline Estabilidad de agregados (\%) & $0-7,5$ & Incremento & $<2$ años & Estacional & Doran et al., 1994 \\
\hline \multicolumn{6}{|l|}{ Indicadores químicos } \\
\hline $\mathrm{pH}$ & $0-7$ & Neutralidad & $>5$ años & Estacional & $\begin{array}{c}\text { Doran et al., } 1994 \\
\text { Arshad y Coen, } 1992\end{array}$ \\
\hline Conductividad eléctrica $\left(\mathrm{dS} \mathrm{m}^{-1}\right)$ & $0-7,5$ & Disminución & $<2$ años & Estacional & Larson y Pierce, 1991 \\
\hline $\mathrm{N}$ total $\left(\mathrm{kg} \mathrm{ha}{ }^{-1} \mathrm{~N}\right)$ & $0-30$ & Incremento & $<2$ años & Estacional & Larson y Pierce, 1991 \\
\hline $\begin{array}{l}\text { Capacidad de intercambio catiónico } \\
\left(\mathrm{cmol}_{\mathrm{c}} \mathrm{kg}^{-1}\right)\end{array}$ & $0-30$ & Incremento & 0 - 10 años & Anual & Arnold et al., 1990 \\
\hline \multicolumn{6}{|l|}{ Indicadores biológicos } \\
\hline Carbono orgánico (Mg C ha-1) & $0-30$ & Incremento & $>10$ años & Anual & Arnold et al., 1990 \\
\hline $\mathrm{N}$ mineralizable $\left(\mathrm{kg} \mathrm{ha}^{-1} \mathrm{~N}\right)$ & $0-30$ & Incremento & $<2$ años & Periódicamente & Doran et al., 1994 \\
\hline Respiración de suelo $\left(\mathrm{kg} \mathrm{C}^{-} \mathrm{CO}_{2} \mathrm{ha}^{-1} \mathrm{~d}^{-1}\right)$ & $0-5$ & Variable & $<2$ años & Estacional & Visser y Parkinson, 1992 \\
\hline
\end{tabular}

en el contexto agrícola, considerando factores como: manejo orgánico e inorgánico (Fließbach et al., 2007), manejo de residuos y labranza (Sharma et al., 2005; Imaz et al., 2010), rotación de cultivos (Mubarak et al., 2005; Aziz et al., 2011), evaluación de calidad de suelo en áreas mineras (Shukla et al., 2004; Afrifa et al., 2011), aplicación de abonos y quemas (Lee et al., 2006; Okonkwo, 2010), entre otros.

Marinari et al. (2006) evaluaron el impacto de prácticas de producción orgánica sobre la calidad de suelo para zonas del centro de Italia, midiendo indicadores microbiológicos y químicos de suelos manejados convencional y orgánicamente, para así, poder comparar su calidad a nivel de finca. Un amplio conjunto de indicadores se seleccionaron en este estudio, dentro de los cuales se encuentra el contenido total de carbono orgánico, nitrógeno orgánico total, contenido disponible de nitrógeno amoniacal y nítrico, conductividad eléctrica y contenido de fósforo disponible. Suelos bajo manejo orgánico mostraron mejores condiciones nutricionales y biológicas, con incrementos en el contenido total de nitrógeno, nitrato y fósforo disponible; no obstante, se encontró que luego de años de prácticas de manejo contrastantes de suelo, no se evidenciaron diferencias consistentes en el contenido de carbono orgánico. Los mayores contenidos de nitrato y fósforo en suelos con manejo orgánico se presentaron como consecuencia de una mayor actividad de las poblaciones de bacterias nitrificantes, siendo finalmente estos dos aspectos los indicadores más aptos para determinar la calidad de los suelos del estudio dada su sensibilidad frente al manejo considerado.

Diferentes técnicas de análisis de datos se han usado para evaluar la calidad de suelos, entre estas, regresión lineal y distintas técnicas multivariantes para el desarrollo de índices (Zornoza et al., 2007). La regresión lineal múltiple ha sido usada por diferentes autores para evaluar la calidad de suelo en bosques naturales y en suelos agrícolas bajo diferentes prácticas de manejo (Lentzsh et al., 2005).

Zornoza et al. (2007) establecieron un modelo utilizando regresión lineal múltiple, basado en diferentes propiedades físicas, químicas y biológicas del suelo, en diferentes zonas forestales de la provincia de Alicante 
(España), consideradas como suelos de excelente calidad. Se analizaron tres suelos Molisoles y un Entisol, desarrollados bajo ambientes de vegetación natural con mínima intervención humana y luego el modelo fue aplicado en dos suelos de avanzado estado de degradación. Se llevó a cabo la regresión lineal integrando diferentes propiedades como textura, capacidad de retención de agua, conductividad eléctrica, fósforo disponible, contenido de carbono soluble, respiración basal de suelo, actividad enzimática y contenido de nitrógeno, carbono orgánico de suelo y biomasa microbiana como parámetros predictores.

Los modelos obtenidos fueron apropiados para reflejar la perturbación sufrida en suelos degradados a partir de la comparación con suelos no disturbados, por lo cual este tipo de técnicas de análisis se constituyen como una herramienta de precisión aceptable para evaluar la calidad de este tipo de suelos.

Pese a los avances en el tema, aún no existe un consenso acerca de los estándares de la calidad de suelos, sobre todo porque ninguna variable biológica y físico-química única o combinada puede reflejar los muchos procesos que interactúan y que son responsables de la calidad del suelo (Puglisi et al., 2006). Aunque las propiedades físicas y químicas se usan ampliamente para evaluar la calidad de este recurso, se ha demostrado que las propiedades basadas en la actividad biológica y bioquímica del suelo, tales como actividad enzimática y estructura de comunidad microbiana, responden a pequeños cambios de las condiciones del suelo, por lo cual proveen información sobre la calidad del suelo luego de sutiles cambios.

Aunque los parámetros químicos en el suelo cambian lentamente, y se requiere de varios años para obtener diferencias significativas (García-Ruíz et al., 2009), ya en 2006, Shukla et al. mostraron que el contenido de carbono orgánico en suelo fue más sensible que parámetros microbiológicos para el monitoreo de calidad de suelo bajo labranza tradicional versus labranza de conservación en suelos franco limosos de Ohio, USA. Quin et al. (2010) probaron si los contenidos de carbono orgánico del suelo, capacidad de intercambio catiónico, $\mathrm{Ca}^{2+}$ y $\mathrm{Mg}^{2+}$ intercambiables, nitrógeno total, fósforo disponible, biomasa microbiana y la actividad de ciertas enzimas en suelo pueden discriminar diferentes prácticas de labranza, y determinaron el indicador más sensible para el monitoreo de calidad en suelos Cambisoles franco limosos. Se obtuvo que todos los parámetros analizados, excepto la capacidad de intercambio catiónico y el contenido de cationes de cambio, fueron significativamente mayores en suelos bajo labranza de reducida (corte y esparcimiento de tallos de maíz luego de la cosecha y preparación superficial de suelo) en comparación con labranza tradicional (recolección de tallos de maíz luego de la cosecha y preparación profunda de suelo). La actividad enzimática y la biomasa microbiana de suelo, fueron los parámetros de mayor sensibilidad para evaluar los efectos de la labranza tradicional vs. la labranza reducida sobre la calidad de suelo.

Entre las distintas prácticas de manejo de suelos en agroecosistemas, la incorporación de enmiendas orgánicas y fertilizantes minerales puede tener el mayor impacto sobre la fertilidad del suelo y el estado de la calidad del mismo. Giacometti et al. (2013) examinaron la interrelación entre indicadores químicos y microbiológicos y su capacidad para diferenciar parcelas bajo regímenes contrastantes de fertilización. Las propiedades seleccionadas demostraron ser importantes indicadores de los cambios a largo plazo de la calidad del suelo y estuvieron fuertemente interrelacionados, particularmente los cambios en el contenido de materia orgánica y su composición inducidos por la modificación de las fuentes de fertilización estuvieron acompañados por cambios en la abundancia y la funcionalidad de la comunidad microbiana del suelo.

Las quemas es otra de las prácticas que pueden perturbar significativamente las propiedades de los suelos, dando lugar a su degradación. Dentro de este marco, la caracterización de estas alteraciones es fundamental para el diseño de estrategias de manejo posquema (Parsons et al., 2010). Neary et al. (2005) afirman que las quemas severas reducen el contenido de carbono orgánico del suelo (COS), no obstante, Hatten y Zabowski (2010) aseguran que no se presenta ningún cambio en este parámetro luego de una quema. La falta de uniformidad en la respuesta de COS sugiere que la relación entre este aspecto y la severidad de la quema de suelo no ha sido definida adecuadamente. Por otro lado, Úbeda y Outeiro (2009) exponen que hay un incremento del pH del suelo como efecto de la quema, lo cual sugiere que esta propiedad es sensible a los cambios de la severidad de la quema de suelos.

Los aumentos en las formas disponibles de $\mathrm{Ca}, \mathrm{Mg}$ y $\mathrm{K}$ inmediatamente después de incendios forestales se han observado en muchos estudios (Neary et al., 2005). Se ha detectado el aumento de la concentración de P disponible en suelos quemados según Kennard y Gholz (2001). Por tanto, vale la pena explorar si los 
cambios en el contenido de estos nutrientes pueden proporcionar información útil para la caracterización de los efectos de la quema de suelos.

Vega et al. (2013), determinaron si algunos signos visuales de severidad de la quema de suelos, reconocidos en campo, están relacionados con cambios en algunas propiedades químicas y biológicas del suelo. Ocho áreas afectadas por incendios forestales en España fueron seleccionadas para el estudio y se analizaron propiedades químicas y biológicas como $\mathrm{pH}, \mathrm{Ca}, \mathrm{K}$, $\mathrm{Mg}$ y $\mathrm{P}$ extractables, nitrógeno total, $\mathrm{NH}_{4}{ }^{+}, \mathrm{NO}_{3}$ extractables, tasa de amonificación y nitrificación y mineralización potencial de $\mathrm{N}$, en relación a cinco niveles de severidad de quema de suelo. Los niveles de severidad de la quema reflejaron adecuadamente los cambios en COS, pH y la actividad enzimática. El estudio demostró que las categorías de severidad propuestas, basadas en signos visuales, son útiles para inferir el grado de degradación de propiedades químicas y microbiológicas, en este caso el carbono orgánico, el pH y la actividad de la fosfatasa ácida, reflejando a su vez cambios en la calidad de suelos quemados.

Evidentemente se observan varios indicadores químicos de calidad de suelo, sin embargo, la pregunta es ¿̇cuál o cuáles de estos indicadores son los más apropiados para la caracterización de los impactos naturales y antrópicos sobre la funcionalidad del suelo? (Schoenholtz et al., 2000; Bertini et al., 2014). Algunos enfoques estadísticos, especialmente técnicas multivariantes como el análisis jerárquico y el análisis de redundancia, se pueden utilizar para seleccionar variables apropiadas para la evaluación de la calidad del suelo.

Bertini et al. (2014) emplearon estos métodos estadísticos para encontrar indicadores de calidad del suelo, utilizando datos microbiológicos, químicos y físicos para comparar dos tipos de bosques (nativos y reforestados) en dos ubicaciones en el sureste de Brasil. El análisis de redundancia mostró que la actividad de la fosfatasa ácida y de la deshidrogenasa, el pH, el contenido de fosforo disponible y el contenido de arenas se correlacionaron positivamente con el bosque nativo, mientras que variables como el carbono orgánico total, el contenido de azufre y de arcilla, se correlacionaron positivamente con el bosque reforestado. Estos resultados sugieren que estas variables son los mejores indicadores de calidad de suelos en las condiciones del estudio. El análisis jerárquico resultó ser una herramienta valiosa y confiable en la determinación de indicadores para evaluar calidad de suelos, que puede usarse en distintas condiciones geográficas y edafológicas.

Jamioi et al. (2015) evaluaron varios indicadores químicos para determinar la calidad de suelo en sistemas productivos de los Llanos Orientales de Colombia. Para el efecto, se incluyeron once sistemas ubicados en terrazas altas, medias y aluviones recientes. En la terraza alta se seleccionó sorgo dulce (Sorghum vulgare), palma de aceite (Elaeis guineensis), soya (Glycine max), piña Golden (Ananas sativus), maíz (Zea mays), y naranja Valencia (Citrus sinensis Osbeck). En la terraza media los sistemas seleccionados fueron: pasto cv. Llanero (Brachiaria dictyoneura), caucho (Hevea brasiliensis), maní forrajero (Arachis pintoi), palma de aceite (Elaeis guineensis), acacia (Acacia mangium), melina (Gmelina arborea) y yopo (Anadenanthera peregrina). En los aluviones recientes se seleccionó pastura degradada de brachiaria (Brachiaria decumbens). Los resultados fueron analizados mediante análisis de componentes principales. El carbono orgánico, el $\mathrm{pH}$, la acidez intercambiable, y los contenidos de calcio, hierro y magnesio fueron las variables priorizadas como indicadores de calidad de suelo. Con esta información se logró proponer una clasificación de la calidad química de los suelos de la región, de gran utilidad al momento de la implementación de sistemas de monitoreo que para la evaluación de la degradación de estos suelos.

Farfán e Hincapié (2011) valoraron la sostenibilidad ambiental haciendo uso de indicadores de calidad de suelo en cultivos de café de Caldas, Cauca y Santander. En Santander se evaluaron café con sombrío de guamo (Inga spuria) + carbonero (Calliandra pittieri), con y sin fertilización, y café con sombrío de guayacán (Guaiacum officinale) + nogal (Juglans regia), con y sin fertilización, y un testigo (lote en barbecho); en Cauca se evaluaron sistemas de producción de café orgánico y convencional, y en Caldas un sistema de producción de café orgánico, dos de café convencional, con y sin aplicación de fertilizantes químicos. A partir de variables físicas, químicas y biológicas se construyó el Índice General de Sostenibilidad Ambiental (IGSA), con el que se concluyó que en Santander los sistemas de producción de café con sombrío de guamo y carbonero, con y sin fertilización, y café con sombrío de guayacán y nogal, con y sin fertilización, son sostenibles, y el testigo se ubicó en la categoría de medianamente sostenible. En Cauca, los sistemas de producción de café orgánico y los convencionales se clasifican en la categoría de sostenibles. En Caldas, los sistemas de producción de café orgánico, 
convencionales y el testigo, se clasifican en la categoría de sostenibles.

\section{CONCLUSIONES}

Dado que la influencia de las prácticas de manejo de los sistemas agrícolas sobre la degradación del suelo, ha aumentado la preocupación por la sostenibilidad de este recurso natural (Oliveira et al., 2016), es esencial armonizar la utilización del recurso suelo y su conservación, mediante la integración de la evaluación de calidad del suelo (Tóth et al., 2007). De ahí se desprende la necesidad de desarrollar métodos fiables y prácticos para evaluar la calidad del recurso, que involucren no solamente propiedades químicas sino también físicas y biológicas, que evidentemente tengan en cuenta la interrelación funcional de estos atributos y su dinámica en función de los usos del suelo.

Los indicadores mencionados, son de gran utilidad para el monitoreo del efecto de los sistemas de uso de suelo sobre algunas de sus propiedades químicas, lo cual es esencial para el diseño de sistemas productivos sostenibles que maximicen lo efectos positivos de las prácticas agrícolas implementadas. Muchos de los indicadores que se tuvieron en cuenta para este estudio, resultan aptos para monitorear la calidad química del suelo, adicionalmente, son fáciles de medir y sensibles a cambios en los sistemas de manejo. A partir de los resultados obtenidos se observa que las propiedades químicas en interacción con el funcionamiento metabólico de las poblaciones microbianas en el suelo son de gran utilidad para estimar la condición del suelo en condiciones específicas de uso.

Conflicto de intereses: el manuscrito fue preparado y revisado con la participación de los autores, quienes declaran no tener algún conflicto de interés que coloquen en riesgo la validez de los resultados aquí presentados.

\section{REFERENCIAS BIBLIOGRÁFICAS}

Afrifa, E.K., F.A. Armah, A.N.M. Pappoe y P.K. Essandoh. 2011. Assessment of the quality of revegetated soil near an active goldmine in southwestern Ghana. Int. J. Environ. Sci. 2, 873-888.

Arnold, R.W., I. Szabolcs y V.O. Targulian. 1990. Global soil change. Report of an International Institute for Applied System Analysis. ISSS, UNEP, Laxenburg, Austria.
Arshad, M.A. y G.M. Coen. 1992. Characterization of soil quality: physical and chemical criteria. Am. J. Alternat. Agr. 7, 25-31. Doi: 10.1017/S0889189300004410

Arshad, M. y S. Martin. 2002. Identifying critical limits for soil quality indicators in agro-ecosystems. Agric. Ecosyst. Environ. 88, 153-160. Doi: 10.1016/ S0167-8809(01)00252-3

Astier, M., M. Maass y J. Etchevers. 2002. Derivación de indicadores de calidad de suelos en el contexto de la agricultura sustentable. Agrocienc. 36, 605-620.

Aziz, I., M. Ashraf, T. Mahmood, and K.R. Islam. 2011. Crop rotation impact on soil quality. Pak. J. Bot. 43(2), 949-960.

Bertini, S.C.B., L.C.B. Azevedo, I. de Carvalho Mendes y E.J.B.N. Cardoso. 2014. Hierarchical partitioning for selection of microbial and chemical indicators of soil quality. Pedobiol. 57(4-6), 293-301. Doi: 10.1016/j. pedobi.2014.06.001

Brejda, J.J., T.B. Moorman, D.L. Karlen y T.H. Dao. 2000. Identification of Regional Soil Quality Factors and Indicators. I. Central and Southern High Plains. Soil Sci. Soc. Am. J. 64, 2115-2124. Doi: 10.2136/ sssaj2000.6462115x

Cantú, M., A. Becker, J. Bedano y H. Schiavo. 2007. Evaluación de la calidad de suelos mediante el uso de indicadores e índices. Cienc. Suelo 25, 173-178.

Chappell, N.A., J.L. Ternan y K. Bidin. 1999. Correlation of physicochemical properties and sub-erosional landforms with aggregate stability variations in a tropical Ultisol disturbed by forestry operations. Soil Till. Res. 50, 55-71. Doi: 10.1016/S0167-1987(98)00196-2

Cruz, B., J.E. Barra, R.F. Castillo y C. Gutiérrez. 2004. La calidad del suelo y sus indicadores. Ecosist. 13, 90-97.

De la Rosa, D. y R. Sobral. 2008. Soil quality and methods for its assessment. Soil Sci. 167-200. Doi: 10.1007/978-1-4020-6778-5_9

Doran, J.W., D.C. Coleman, D.C. Bezdicek y B.A. Stewart. 1994. Defining and assessing soil quality for sustainable environment. Soil Science Society of America. Special Publication 35. Madison, Wisconsin, USA.

Farfán, F. y E. Hincapié. 2011. Valoración de la sostenibilidad ambiental mediante indicadores de calidad del suelo, en sistemas de producción de café en Colombia. Cenicafé 62(1), 100-118.

Franzluebbers, A.J. y M.A. Arshad. 1996. Water-stable aggregation and organic matter in four soils under conventional and zero tillage. Can. J. Soil Sci. 76, 387-393. Doi: 10.4141/cjss96-046

Fliebach, A., H.R. Oberholzer, L. Gunst y P. Mader. 2007. Soil organic matter and biological soil quality indicators after 21 years of organic and conventional farming. Agric. Ecosyst. Environ 118, 273-284. Doi: 10.1016/j.agee.2006.05.022 
García, Y., W. Ramírez y S. Sánchez. 2012. Indicadores de la calidad de los suelos: una nueva manera de evaluar este recurso. Pastos Forrajes 35, 125-137.

García-Ruiz, R., V. Ochoa, B. Vi-egla, M.B. Hinojosa, R. Pe-a-Santiago, G. Liébanas, J. Linares y J.A. Carreira. 2009. Soil enzymes, nematode community and selected physico-chemical properties as soil quality indicators in organic and conventional olive oil farming: Influence of seasonality and site features. Appl. Soil Ecol. 41, 305-314. Doi: 10.1016/j.apsoil.2008.12.004

Giacometti, C., M.S. Demyan, L. Cavani, C. Marzadori, C. Ciavatta y E. Kandeler. 2013. Chemical and microbiological soil quality indicators and their potential to differentiate fertilization regimes in temperate agroecosystems. Appl. Soil Ecol. 64, 32-48. Doi: 10.1016/j. apsoil.2012.10.002

Govaerts, B., K.D. Sayre y J. Deckers. 2006. A minimum data set for soil quality assessment of wheat and maize cropping in the highlands of Mexico. Soil Till. Res. 87, 163-74. Doi: 10.1016/j.still.2005.03.005

Hatten, J.A. y D. Zabowski. 2010. Fire severity effects on soil organic matter from a ponderosa pine forest: a laboratory study. Int. J. Wildland Fire 19, 613-623. Doi: 10.1071/WF08048

Hünnemeyer, J.A., R. De Camino y S. Müller. 1997. Análisis del desarrollo sostenible en centroamérica: Indicadores para la agricultura y los recursos naturales. IICA/ GTZ, San José, Costa Rica.

Imaz, M.J., I. Virto, P. Bescansa, A. Enrique, O. Fernandez-Ugalde y D.L. Karlen. 2010. Soil quality indicator response to tillage and residue management on semi-arid Mediterranean cropland. Soil Till. Res. 107, 17-25. Doi: 10.1016/j.still.2010.02.003

Jamioi, D.D., J.C. Menjivar y Y. Rubiano. 2015. Indicadores químicos de calidad de suelos en sistemas productivos del Piedemonte de los Llanos Orientales de Colombia. Acta Agron. 64(4), 302-307.

Karlen, D.L., S.S. Andrews, B.J. Weinhold y J.W. Doran. 2003. Soil quality: Humankind's foundation for survival. J. Soil Water Conserv. 58(4), 171-179.

Karlen, D.L., M.J. Mausbach, J.W. Doran, R.G. Cline, R.F. Harris y G.E. Schuman. 1997. Soil quality: a concept, definition, and framework for evaluation. Soil Sci. Soc. Am. J. 61, 4-10. Doi: 10.2136/ sssaj1997.03615995006100010001x

Karlen, D.L., S.S. Andrews, B.J. Wienhold y T.M. Zobeck. 2008. Soil quality assessment: past, present and future. J. Int. Biosc. 6, 3-14.

Kennard, D.K. y H.L. Gholz. 2001. Effects of high- and lowintensity fires on soil properties and plant growth in a Bolivian dry forest. Plant Soil 234, 119-129. Doi: 10.1023/A:1010507414994

Khormali, F. y S. Shamsi. 2009. Micromorphology and quality attributes of the loess derived soils affected by land use change: A case study in Ghapan watershed, Northern Iran. J. Mt. Sci. 6, 197-204. Doi: 10.1007/ s11629-009-1037-z

Lal, R. 1998. Soil quality and agricultural sustainability. Ann Arbor Press, Chelsea, MI, USA.

Larson, W. E. y F.J. Pierce. 1991. Conservation and enhancement of soil quality. pp. 175-203. En: Evaluation for sustainable land management in the developing world. Vol. 2. IBSRAM Proc. 12(2). International Board for Soil Resources and Management. Bangkok, Tailandia.

Larson, W.E. y F.J. Pierce. 1994. The dynamics of soil quality as a measure of sustainable management. pp. 3752. En: Coleman, D.C., D.F. Bezdicek y B.A. Stewart (eds.). Defining soil quality for a sustainable environment. Sol. Sci. Soc. Am., Special Publication No. 35. SSSA, Madison, WI, USA.

Lee, C.H., M.Y. Wu, V.B. Asio y Z.S. Chen. 2006. Using a soil quality index to assess the effects of applying swine manure compost on soil quality under a crop rotation system in Taiwan. Soil Sci. 171, 210-222. Doi: 10.1097/01.ss.0000199700.78956.8c

Lentzsh, P., R. Wieland y S. Wirth. 2005. Application of multiple regression and neural network approaches for landscape-scale assessment of soil microbial biomass. Soil Biol. Biochem. 37, 1577-1580. Doi: 10.1016/j. soilbio.2005.01.017

Marinari, S., R. Mancinelli, E. Campiglia y S. Grego. 2006. Chemical and biological indicators of soil quality in organic and conventional farming systems in Central Italy. Ecol. Indic. 6(4), 701-711. Doi: 10.1016/j. ecolind.2005.08.029

Mubarak, A.R., O.M.E. Elshami y A.A. Azhari. 2005. Longand short-term effects of cultivation on properties of a Vertisol under sugarcane plantation. Soil Till. Res. 84, 1-6. Doi: 10.1016/j.still.2004.08.005

Neary, D.G., K.C. Ryan y L.F. DeBano. 2005. Wildland fire in ecosystems. Effects of fire on soil and water. USDA Forest Service, Rocky Mountain Research Station. General Technical Report RMRS-GTR-42-vol 4, Ogden, UT, USA.

Okonkwo, C.I. 2010. Effect of burning and cultivation on soil properties and microbial population of four different land use systems in Abakaliki. Res. J. Agric. Biol. Sci. 6(6), 1007-1014.

Oliveira, S.P., D. Cândido, M.J. Weber, O.B. Xavier, F.A.S. Escobar y T.S. Oliveira. 2016. Conversion of forest into irrigated pasture I. Changes in the chemical and biological properties of the soil. Catena 137, 508-516. Doi: 10.1016/j.catena.2015.10.017

Parsons, A., P. Robichaud, S. Lewis, C. Napper y J. Clark. 2010. Field guide for mapping post-fire soil burn 
severity. USDA For. Serv. Gen .Tech. Rep. RMRSGTR-243. Washington D.C., USA.

Parr, J.F. y R.I. Papendick. 1997. Soil quality: relationship and strategies for sustainable dryland farming system. Ann. Arid Zone 36, 181-191.

Puglisi, E., A.A.M. Del Re, M.A. Rao y L. Gianfreda. 2006. Development and validation of numerical indexes integrating enzyme activities of soils. Soil Biol. Biochem. 38, 1673-1681. Doi: 10.1016/j.soilbio.2005.11.021

Schoenholtz, S.H., H.V. Miegroet y J.A. Burger. 2000. A review of chemical and phys- ical properties as indicators of forest soil quality: challenges and opportunities. For. Ecol. Manag. 138, 335-356. Doi: 10.1016/ S0378-1127(00)00423-0

Sharma, K.L., U.K. Mandal, K. Srinivas, K.P.R. Vittal, B. Mandal, J.K. Grace y V. Ramesh. 2005. Long-term soil management effects on crop yields and soil quality in a dryland Alfisol. Soil Till. Res. 83, 246-259. Doi: 10.1016/j.still.2004.08.002

Shukla, M.K., R. Lal y M. Ebinger. 2006. Determining soil quality indicators by factor analysis. Soil Till. Res. 87, 194-204. Doi: 10.1016/j.still.2005.03.011

Shukla, M.K., R. Lal y M. Ebinger. 2004. Soil quality indicators for reclaimed minesoils in southeastern Ohio. Soil Sci. 169, 133-142. Doi: 10.1097/01. ss.0000117785.98510.0f

Soil Quality Institute (SOI). 1996. Indicators for soil quality evaluation. USDA Natural Resources Conservation Service, Washington D.C., USA.

Tóth, G., V. Stolbovoy y L. Montanarella. 2007. Soil Quality and sustainability evaluation - An integrated approach to support soil-related policies of the European Union.
Eur 22721. Office for Official Publications of the European Communities, Luxembourg.

Úbeda, X. y L. Outeiro. 2009. Physical and chemical effects of fire on soil. pp. 105-132. En: Cerdà, A. y P.R. Robichaud (eds.). Fire effects on soils and restoration strategies. Science Publishers, Estfield, NH, USA. Doi: 10.1201/9781439843338-c4

Vega, J.A., T. Fontúrbel, A. Merino, C. Fernández, A. Ferreiro y E. Jiménez. 2013. Testing the ability of visual indicators of soil burn severity to reflect changes in soil chemical and microbial properties in pine forests and shrubland. Plant Soil 369(1-2), 73-91. Doi: 10.1007/ s11104-012-1532-9

Visser, S. y D. Parkinson. 1992. Soil biological criteria as indicators of soil quality. Soil Microorg. 7, 25-31.

Vose, J.M., W.T. Swank, B.D. Clinton, J.D. Knoepp y L.W. Swift. 1999. Using stand replacement fires to restore southern Appalachian pine-hardwood ecosystems: effects on mass, carbon, and nutrient pools. For Ecol. Manag. 114, 215-226. Doi: 10.1016/ S0378-1127(98)00352-1

Yao, R., J. Yang, P. Gao, J. Zhang y W. Jin. 2013. Determining minimum data set for soil quality assessment of typical salt-affected farmland in the coastal reclamation area. Soil Till. Res. 128, 137-148. Doi: 10.1016/j. still.2012.11.007

Zornoza, R., J. Mataix-Solera, C. Guerrero, V. Arcenegui, F. García-Orenes, J. Mataix-Beneyto y A. Morugán. 2007. Evaluation of soil quality using multiple lineal regression based on physical, chemical and biochemical properties. Sci. Total Environ. 378(1-2), 233-237. Doi: 10.1016/j.scitotenv.2007.01.052 